\title{
O Desafio da Avaliação na Formação Médica por Competência
}

\author{
Eliane Dias Gontijo ${ }^{1}$ \\ Cristina Gonçalves Alvim ${ }^{1}$ \\ Zilma S. Nogueira Reis ${ }^{1}$ \\ ${ }^{1}$ Universidade Federal de Minas Gerais, UFMG, Brasil; E-Mail: egontijo@medicina.ufmg.br; \\ cristinagalvim@gmail.com; zilma.medicina@gmail.com
}

\section{Resumo}

As Diretrizes Curriculares Nacionais brasileiras do Curso de Graduação em Medicina direcionam recomendações para as instituições de ensino superior, com foco na formação baseada no desenvolvimento de competências. Tal apontamento implica em desenvolver no discente a capacidade de mobilizar não apenas conhecimentos, mas também habilidades e atitudes para lidar com situações e dilemas reais, preparando-o para o cenário de atuação profissional do médico no país. Os autores apresentam um novo modelo conceitual, integrativo, não hierárquico e representado por um átomo, cujos atributos: saber, aplicar, demonstrar, fazer, ser e interagir orbitam em torno do desenvolvimento de competências essenciais. Tal concepção, uma remodelação da tradicional pirâmide proposta por Miller, é fruto das experiências avaliativas desenvolvidas na instituição, de forma a favorecer a reflexão sobre a complexidade do processo da formação médica.

Palavras-chave: avaliação educacional; competência clínica; educação baseada em competências; educação médica.

\begin{abstract}
The Brazilian National Curriculum Guidelines for Undergraduate Medicine Courses indicates recommendations for higher education institutions, in the context of competence- based approach. Such approach implies developing in the medicine students the capacity to mobilize, not only knowledge but also expertise and attitudes to deal with real situations and dilemmas, preparing them for the scenario of professional practice of physician's in the country. The authors present a new conceptual model, not hierarchical and represented by an atom, whose attributes: knowing, applying, demonstrating, doing, being and interacting orbited around developing the core competencies. This conception, a remodeling of the traditional pyramid model proposed by Miller, is the result of the evaluative experiences developed in the institution, to favor reflection on the complexity of the medical training process..
\end{abstract}

Keywords: clinical competence; competency-based education; educational measurement; education medical.

\section{Formação Médica na Contemporaneidade}

O processo de formação médica vincula-se às demandas e necessidades de saúde, às políticas públicas de educação e saúde, e às formas de organização dos serviços. Na sociedade contemporânea, tende-se a valorizar mais o olhar instrumentado do que o olhar clínico, acarretando a fragmentação do cuidado e dos saberes.

Docentes especialistas pressionam pela introdução de conteúdos ou até novas disciplinas nos cursos, motivados em permanecer no conforto de sua expertise, em práticas de ensino-aprendizagem centradas no professor, com base em uma relação teoria-prática que posterga o exercício de integração e articulação de saberes e fazeres.

Apesar do amplo reconhecimento dos múltiplos determinantes do processo saúde-doença e a influência inequívoca de fatores não biológicos, a abordagem biológica e curativa, ainda, predomina na formação médica. Negligencia-se, além disso, o fato de a clínica ser sempre uma relação entre dois sujeitos, irredutível à objetividade, e influenciada pelas histórias e subjetividade desses dois elementos (Almeida, Feuerwerker, \& Llanos, 1999). Em 1998, o Relatório 
Delors - Unesco apontou a necessidade da formação holística do indivíduo e a aprendizagem ao longo da vida (Delors et al., 1998). Considera que a prática pedagógica deve desenvolver quatro aprendizagens fundamentais, que seriam para cada indivíduo os pilares do conhecimento:

aprender a conhecer indica o interesse, a abertura para a gestão do conhecimento, de modo a impedir que as pessoas fiquem ilhadas pelo número de informações, mais ou menos efêmeras, que invadem os espaços públicos e privados; aprender a fazer para agir com responsabilidade; aprender a conviver traz o desafio do respeito e cooperação com os outros; e, finalmente, aprender a ser, que ao explicitar o papel do cidadão, integra todos os demais (Delors et al., 1998).

Entretanto, verifica-se que o ensino médico atual, ainda, se apoia apenas em um dos pilares: aprender a conhecer, e em menor escala, no aprender a fazer. Os outros dois ou são negligenciados, ou são subentendidos como consequências naturais dos primeiros e nem sequer são avaliados.

As Diretrizes Curriculares Nacionais brasileiras para o curso de Medicina preconizam uma formação baseada no desenvolvimento de Competências (Brasil, 2014). O ensino por competências implica em desenvolver no estudante a capacidade de mobilizar os conhecimentos, habilidades e atitudes para lidar com situações, problemas e dilemas da vida real (Perrenoud, 1999). As demandas de saúde da população não se apresentam como estruturas rígidas; ao contrário, os problemas são complexos e imprecisos. Assim, os estudantes devem aprender a tomar decisões sob condições de incerteza, a lidar com a ambiguidade, com a complexidade, a singularidade e os conflitos de valores que quase sempre escapam à racionalidade técnica. No entanto, o movimento inovador dentro de uma instituição de ensino médico é tão importante quanto as diretrizes nacionais (Cyrino \& Toralles-Pereira, 2004). Ao valorizar transformações a partir das experiências e reflexões de seu corpo docente, a instituição se posiciona de forma ativa, como agente das mudanças na formação. Vocacionado para o cuidado e inovação em saúde, e atuando em múltiplos papeis voltados para o ensino, pesquisa e extensão universitária, o professor contribui também para a discussão dos modelos de ensino e de avaliação do estudante.

Camargo Jr (1992) considera o ato médico como expressão concreta do encontro, na prática clínica, do sofrer do paciente, que leva ao médico seu sofrimento expresso por sintomas, o qual, por sua vez, utiliza seu saber, pretensamente neutro, para diagnosticar e tratar (Camargo Jr, 1992). Bastos (2006) discute a prática da medicina como Ciência e Arte e aponta pontos de tensão entre a objetividade requerida pela ciência e a subjetividade inerente à prática clínica, que podem resultar em insatisfações tanto para pacientes quanto para médicos (Bastos, 2006). Para a autora, a arte acontece quando o médico lida com a singularidade e utiliza sua sensibilidade e intuição para aplicar seu conhecimento, e conclui que na relação médico-paciente, se aspectos emocionais são considerados, a prática médica se humaniza, tornando-se ética.

Outro aspecto relevante da formação médica contemporânea se refere à incorporação de novas tecnologias. A Faculdade de Medicina da UFMG tem acumulado experiências institucionais e estimulado o uso do ensino mediado por tecnologia, em um movimento denominado Ambiente Virtual de Aprendizagem em Saúde para o Século XXI (AVAS21). Trata-se da organização de um espaço virtual, dinâmico o suficiente para permitir que o aluno se organize para estudar, com maior flexibilidade de horários, em relação às atividades presenciais e respeito ao seu ritmo individual. Mesmo a avaliação pode em muito se beneficiar da simulação virtual. O Projeto Imagem da Semana, por exemplo, desde 2012, envolve acadêmicos e professores da Instituição na forma de desafios clínicos semanais publicados na homepage da Faculdade de Medicina, contribuindo com sucesso para a formação profissional e educação continuada (Schützel et al., 2012). Tal modalidade tem sido oferecida por universidades e outras organizações, de forma ampla, incluindo ensino, avaliação e certificação do aprendiz de ensino superior e pósgraduação (Inc, 2016).

\section{A Proposição de um Modelo Integrativo para a Avaliação de Competências}

Um dos grandes desafios das instituições formadoras tem sido garantir para a sociedade, que os egressos de seus cursos se tornaram médicos qualificados, capazes de cuidar e realizar a atenção à saúde integral das pessoas. Essa certificação expressa legitimação social de indivíduos que passam a ser reconhecidos como competentes para atuar na profissão. Nesse sentido, a avaliação do estudante constitui etapa essencial do processo educacional e tem como finalidade monitorar os processos de ensino e, assim, permitir aos docentes e aos estudantes averiguarem se os objetivos educacionais planejados estão sendo desenvolvidos. Além disso, a forma e o conteúdo das avaliações são 
altamente importantes, na medida em que constituem poderoso determinante de como os estudantes estudam e aprendem (Lowry, 1993).

Miller representou um modelo para o processo avaliativo do desempenho discente em formato de pirâmide, composto por quatro níveis pelos quais o estudante deve passar durante sua formação (Miller, 1990). A base referese ao saber, que reporta ao conhecimento dos fatos, princípios e teorias. O segundo nível é o saber como faz, que envolve habilidades de resolver problemas e descrever procedimentos. O próximo nível é o mostrar como faz, com a demonstração de habilidades em situação simulada ou padronizada. O quarto e último nível é o fazer, no qual o estudante é observado em situação de prática com pacientes reais.

Em 2013, a elaboração da matriz de competências essenciais para o curso médico da Universidade Federal de Minas Gerais, Brasil, com o objetivo de valorizar a intencionalidade dos percursos acadêmicos e servir de referência aos processos avaliativos dos estudantes (Gontijo et al., 2013), levou à reflexão de que uma estrutura piramidal, alicerçada em uma ampla base de conhecimentos, tendo o fazer no seu ápice, poderia não ser a melhor representação que norteasse a avaliação do desempenho do estudante em demonstrar sua capacidade de mobilizar conhecimentos, habilidades e valores, de forma integrada. Não é apenas a posse de conhecimentos que avaliza a competência, mas sim o uso que se é capaz de fazer dos conhecimentos frente a situações concretas.

Essa abrangência do conceito de competência médica foi reforçada por Epstein e Hundert (2002):

uso habitual e judicioso de habilidades de comunicação, conhecimento, habilidades técnicas, raciocínio clínico, emoções, valores e reflexão sobre a prática diária para o benefício do indivíduo e da comunidade que está sendo atendida" (Epstein \& Hundert, 2002).

Concordando com Perrenoud que as competências não são por elas mesmas saberes, fazeres ou atitudes, mas a mobilização e integração desses recursos, surge a necessidade de uma representação mais interativa, que articule e integre os diversos atributos, capaz de iluminar os processos de avaliação do desempenho discente (Perrenoud, 1999).

Em Educação Médica, o desenvolvimento de competências é entendido como um eixo estruturante da formação e deve orientar os conteúdos de ensino, as estratégias educacionais e os processos avaliativos. A noção de competência necessita ser explorada em seus limites ainda imprecisos, que compreendem inter-relações entre os atributos cognitivos, psicomotores e afetivos que, conjuntamente, demonstram diferentes modos de realizar, com excelência, uma prática profissional.

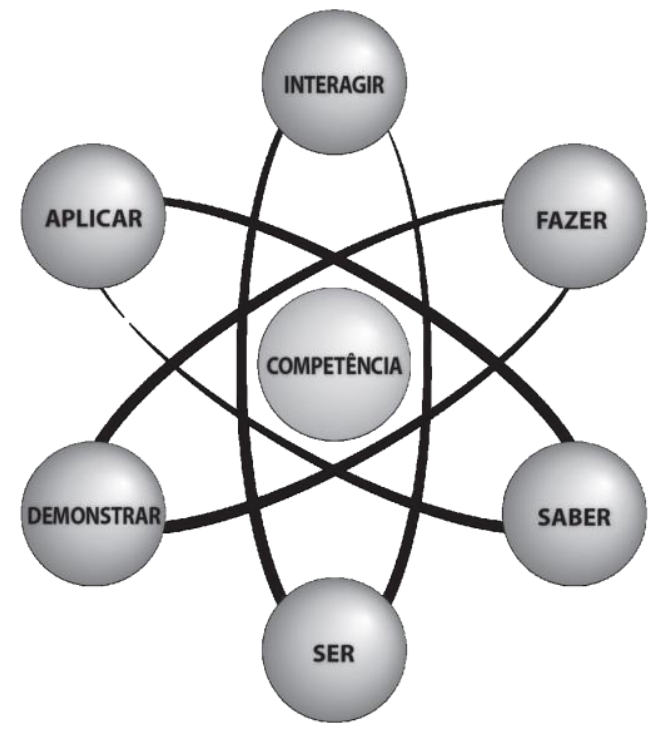

Figura 1. Modelo conceitual em átomo dos atributos da competência médica.

O modelo integrativo aqui proposto (Figura 1), não sendo hierárquico e ao representar os atributos orbitando em torno da competência auxilia na reflexão sobre a complexidade do processo avaliativo na formação médica. Nesse sentido, reforça que avaliar não se limita à verificação de capacidades de forma fragmentada e descontextualizada e 
aponta a necessidade de integrar os diversos atributos, em diferentes contextos na avaliação do desenvolvimento da competência clínica (Gontijo, Alvim, \& de Castro Lima, 2015).

Essa concepção remete ao conceito de Metacognição (Flavell, 1976 apud Ribeiro, 2003) para identificar o que o estudante sabe, o que ele demonstra fazer em situação simulada e, efetivamente, faz em sua prática real, ou seja, avalia a auto percepção sobre os processos cognitivos, elemento essencial para a gestão do conhecimento (Ribeiro, 2003; Flavell, 1976).

O saber - conteúdos disciplinares que abrangem conceitos, teorias, modelos explicativos - deve ser continuamente atualizado e se complementa com a aplicação do conhecimento, em novas situações. A formação e o desenvolvimento de conceitos científicos vão sendo ampliados e aprofundados na medida da incorporação de novas informações, pela oportunidade de revisitá-los em contextos diferentes e de verificar sua aplicação em situações concretas. A aprendizagem significativa, segundo a teoria de Ausubel, acontece quando o que se aprende faz sentido para quem aprende, ou seja, quando novos saberes, valores, emoções, atitudes e habilidades conectam-se à rede de significados, que cada um traz, atribuindo novos sentidos ao fazer (Ronca, 1994).

Incluir a dimensão do significado implica em admitir que o conhecimento não pode ser caracterizado de forma independente de como foi aprendido e de como é usado, pois na medida em que a contextualização necessária para o seu uso o transforma, já não se trata mais do mesmo conhecimento original. Ser capaz de aplicar um conhecimento em um contexto não necessariamente significa sê-lo em qualquer outro; ao contrário, cada contexto significa uma aprendizagem, e em cada experiência transformam-se também as ideias.

Assim, o Saber integra-se com o Fazer. Os alunos, ao se depararem com situações de prática, podem identificar novas formas de abordar a realidade, de estabelecer e testar categorias de compreensão e estratégias de ação. A educação precisa se iniciar a partir de suas demandas e organizar-se de modo que os estudantes aprendam com suas ações - aprender fazendo - e se enriqueçam com as experiências de colegas.

Habilidades de comunicação, técnicas de exame físico, raciocínio e a utilização de recursos diagnósticos e terapêuticos tecnológicos são demandas da formação profissional que se beneficiam da simulação (Troncon, 2007). Em um cenário mundial onde a tecnologia tem posição de destaque, o seu emprego como metodologia ativa de ensino na educação superior pode ser beneficiado, seja na modalidade de ensino à distância, assim como na modalidade presencial (Reis et al., 2016). Constitui-se ainda numa oportunidade de trazer à discussão os exageros no emprego da tecnologia em saúde, pontos positivos, negativos e dilemas éticos quando da sua utilização, em benefícios do cuidado em saúde.

A simulação presencial em ambientes realísticos preparados, usando manequins com e sem robotização, pacientes simulados e teatralização são possibilidades do estudante demonstrar sua habilidade na execução de procedimentos, precedendo o fazer real, garantindo, assim, a segurança do paciente e respeitando preceitos éticos.

O modelo integrativo em átomo aqui apresentado acrescenta dois elementos aos níveis propostos anteriormente por Miller: o Ser e o Interagir. Uma das dimensões da formação do estudante que mais exige atenção na contemporaneidade é o "aprender a ser", traduzido basicamente por formação ética e profissionalismo. O conceito de profissionalismo não é consensual e vem sendo modificado ao longo do tempo (van Mook et al., 2009). Vários elementos compõem as definições atuais de profissionalismo, sendo os mais comuns: compromisso com a excelência, integridade, respeito por outros, empatia, responsabilidade, confidencialidade, colaboração, altruísmo, humanismo e honestidade. Saber se auto avaliar e lidar com conflitos, erros e incertezas também são aspectos essenciais do profissionalismo. A competência moral foi definida por Kohlberg como "a capacidade de tomar decisões e fazer julgamentos que são morais, baseados em princípios internos, e de agir de acordo com tais julgamentos" (Feitosa et al., 2013). Além dos conhecimentos e das habilidades técnicas, o estudante precisa compreender, refletir sobre e aplicar os valores morais que norteiam a boa prática médica.

O interagir foi destacado no modelo proposto para ressaltar a importância das interações sociais no exercício da Medicina, tanto na relação médico-paciente como na relação entre os profissionais e com a sociedade. A formação para a interação com pessoas merece receber um cuidado maior em tempos de digitalização da vida social. A mediação da comunicação por novas tecnologias precisa ser analisada em suas implicações positivas e negativas, como por exemplo, o uso de computadores e celulares durante a aula ou consulta médica e seu efeito sobre a atenção e a empatia. Assim, o ser se complementa com o interagir, constituindo a base para relações interpessoais compreensivas e colaborativas. 
Tendo como referência o modelo integrativo, é possível realizar o feedback do estudante, contemplando todos os aspectos essenciais de sua formação. 0 momento do feedback é uma oportunidade para identificar fortalezas e fragilidades no processo formativo, assim como elaborar propostas para o seu aprimoramento. Professor e estudante reconhecem o que está bom/adequado e o que precisa melhorar, a partir de uma interação dialógica, e propõem estratégias de correção.

O modelo integrativo em átomo reforça a essência da formação de alunos como protagonistas de seu aprendizado, conscientes e responsáveis pelas pessoas e enfatiza a relevância de aprenderem a arte de ser médicos, superando obstáculos, gerenciando conflitos, enfim, aliando competências técnicas com valores e atitudes profissionais.

\section{Referências}

Almeida, M., Feuerwerker, L., \& Llanos, C. (1999). Educação dos profissionais de saúde na América Latina: teoria e prática de um movimento de mudança. In Saúde em Debate. Hucitec Lugar Editorial.

Bastos, L. (2002). Corpo e subjetividade na Medicina: impasses e paradoxos. Universidade do Estado do Rio de Janeiro. Instituto de Medicina Social.

Brasil. (2014). Resolução no 3, de 20 de junho de 2014. Institui Diretrizes Curriculares Nacionais do Curso de Graduação em Medicina e dá outras providências. edited by Ministério da Educação. Conselho Nacional de Educação. Câmara de Educação Superior. Brasília: Portal do Ministério da Educação. Retrieved from: Disponível em: http://portal.mec.gov.br/cne/arquivos/pdf/Med.pdf.

Camargo Jr, K. (1992). (Ir) racionalidade médica: os paradoxos da clínica. Physis: Revista de Saúde Coletiva 2(1), 203230.

Cyrino, E., \& Toralles-Pereira, M. L. (2004). Trabalhando com estratégias de ensino-aprendizado por descoberta na área da saúde: a problematização e a aprendizagem baseada em problemas. Cadernos de Saúde Pública 20:780-788.

Delors, J., Tunas Eufrásio, J. C., \& Carneiro, R., and Century International Commission on Education for the TwentyFirst. (1998). Educação: um tesouro a descobrir: relatório para a Unesco da Comissão Internacional sobre Educação para o século XXI.

Epstein, Ronald M., \& Hundert, E. (2002). Defining and assessing professional competence. Jama 287(2), 226-235 \%@0098-7484.

Feitosa, Helvécio Neves., Rego, S., Bataglia, P., Rego, G., \& Nunes, R. (2013). Competência de juízo moral dos estudantes de medicina: um estudo piloto. Revista Brasileira de Educação Médica: 5-14.

Flavell, John H. (1976). Metacognitive aspects of problem solving. The nature of intelligence: 231-235.

Gontijo, E. D., Gonçalves, C. A., \& Lima, M. E. (2015). Manual de avaliação da aprendizagem no curso de graduação em Medicina. Revista Docência do Ensino Superior 5(1), 205-325.

Gontijo, E. D., Alvim, C., Megale, L., Renan, C. J., \& Lima, M. (2013). Matriz de competências essenciais para a formação e avaliação de desempenho de estudantes de Medicina. Rev. Bras Educ Médica 37(4), 526-539.

Inc, Coursera. 2016. Coursera. accessed 28/05/2016. https://www.coursera.org/.

Lowry, Stella. 1993. Assessment of students. BMJ: British Medical Journal 306 (6869), 51.

Miller, George E. (1990). The assessment of clinical skills/competence/performance. Academic medicine 65(9), S637.

Perrenoud, Philippe. 1999. Avaliação: da excelência à regulação das aprendizagens-entre duas lógicas: Artmed.

Reis, Z. S. N., Melo, M. C., Corrêa, E. J., Pereira, A. K., Santos D. B., \& Alves, H. J. (2016). TECNOLOGIAS DIGITAIS PARA O ENSINO EM SAÚDE: relato de experiências e a convergência para o Projeto AVAS21. Revista de Saúde Digital e Tecnologias Educacionais-RESDITE 1(1).

Ribeiro, Célia. (2003). Metacognição: um apoio ao processo de aprendizagem. Psicologia: reflexão e crítica 16 (1), 109-116. 
Ronca, A. C. C. Teorias de ensino: a contribuição de David Ausubel. Temas Psicol [Internet]. (1994) Dec [cited 2015 Feb 22]; 2(3), 91-5.

Schützel, Manuel., Rodrigues, C. J., Sandra Monetti S. M., \& Parisotto, V. S. (2012). Projeto imagem da semana. Revista Brasileira de Educação Médica 36(3), 423-430.

Troncon, L. E. A. (2007). Utilização de pacientes simulados no ensino e na avaliação de habilidades clínicas. Medicina (Ribeirao Preto. Online), 40(2).

van Mook, Walther N. K. A., Luijk, Scheltus J. van, O'Sullivan, Helen, Wass, Valerie, Zwaveling, Jan Harm, Schuwirth, Lambert W., and van der Vleuten, Cees P. M. (2009). The concepts of professionalism and professional behaviour: Conflicts in both definition and learning outcomes. European Journal of Internal Medicine 20 (4), e85-e89.

\section{Sobre os Autores}

Eliane Dias Gontijo é Professora titular do Departamento de Medicina Preventiva e Social. Núcleo de Educação Médica da Faculdade de Medicina da Universidade Federal de Minas Gerais (UFMG), Belo Horizonte, Brasil.

Cristina Gonçalves Alvim é Professora Associada do Departamento de Pediatria. Diretoria de Avaliação Institucional da Universidade Federal de Minas Gerais, Faculdade de Medicina da UFMG.

Zilma S. Nogueira Reis Medicina (PhD) é Professora Associada do Departamento de Ginecologia e Obstetrícia, do Programa de Pósgraduação em Saúde da Mulher da UFMG e Médica do Hospital das Clínicas. Coordena o Centro de Informática Médica da Faculdade de Medicina da UFMG desde 2012. É Vice-presidente da Sociedade Brasileira de Informática em Saúde (SBIS). Médica ginecologista e obstetra (1990), Mestre em Medicina (1993) e Doutora em Medicina (2002) pela Universidade Federal de Minas Gerais, quando obteve bolsa de doutorado-sanduiche na Alemanha, PROBRAL 113/00 (CAPES/DAAD). Realizou Estágio Sênior Pós-Doutoral no Exterior com bolsa CAPES, na Universidade do Porto, Portugal (2011). 